\title{
Vulvar lichen sclerosus: recent advances in understanding and management
}

\author{
Anupma Kumari ${ }^{1 *}$, Pushpendra K. Singh ${ }^{2}$, Renu Rohatgi ${ }^{3}$
}

\author{
${ }^{1}$ Department of Obstetrics and Gynecology, HMCH, Rourkela, Odisha, India \\ ${ }^{2}$ Department of Dermatology, LMH, Muzaffarpur, Bihar, India \\ ${ }^{3}$ Department of Obstetrics and Gynecology, NMCH, Patna, Bihar, India
}

Received: 13 August 2021

Accepted: 06 September 2021

\author{
*Correspondence: \\ Dr. Anupma Kumari, \\ E-mail anupma.anmmch@gmail.com
}

Copyright: (c) the author(s), publisher and licensee Medip Academy. This is an open-access article distributed under the terms of the Creative Commons Attribution Non-Commercial License, which permits unrestricted non-commercial use, distribution, and reproduction in any medium, provided the original work is properly cited.

\begin{abstract}
Vulvar lichen sclerosus (VLS) is a chronic inflammatory disorder, which affects women of all ages. It is one of the most common pathologies presenting to vulvar clinics. However, uncertainty continues to exist about its etiopathogenesis, diagnosis and treatment. Studies suggest a multifactorial origin as far as etiology is concerned, including a genetic, autoimmune, hormonal and local infectious background. There is often a delay in diagnosis of VLS due to its asymptomatic nature and lack of awareness in patients as well as physicians. Embarrassment of patients due to private nature of the disease and failure to examine the genital skin properly are the other reasons for delay in diagnosis. Conventionally, treatment includes topical corticosteroids as a first-line therapy with alternative options such as topical calcineurin inhibitors, topical and systemic retinoids, other steroid creams, various destructive techniques and surgical removal of affected tissues. New therapeutic approaches are coming into effect in gynecological practice due to potential risks of the above-mentioned methods. Stem cell and platelet-rich plasma therapy, energy-based modalities such as the fractional $\mathrm{CO}_{2}$ laser, photo dynamic therapy, and high intensity focused ultrasound, and new topical medicines, are some of the new options applied to improve the efficacy of treatment avoiding the side effects of conventional methods. Refinement of surgical techniques for restoring vulvar anatomy is leading to improved patient outcomes. This review summarizes current perspectives on the etiopathogenesis, diagnosis and treatment for vulvar lichen sclerosus.
\end{abstract}

Keywords: Vulvar lichen sclerosus, Review, Etiopathogenesis, Diagnosis, Treatment

\section{INTRODUCTION}

Lichen sclerosus (LS) is a chronic, inflammatory, cutaneous disorder that can lead to scarring, impaired sexual function, and malignancy. LS can affect any area of the body of both males and females, it has a predilection for female anogenital epithelium. ${ }^{1}$ Vulvar lichen sclerosus (VLS) is one of the most common pathologies presenting to vulvar clinics. $13 \%$ of women present with symptomatic vulvar disease. ${ }^{2}$ As VLS causes substantial discomfort (intractable itching, soreness, constipation, and dyspareunia) and morbidity (introital narrowing, burying of the clitoris, and atrophy labia minora), quality of life can be greatly impaired, and patients suffer psychologically and physically. ${ }^{3}$

Diagnosis of VLS is made from the history, typical appearance of lesions, and histology. However, time from reported onset of symptoms until diagnosis may range from 5 to 15 years. This suggests that this condition is commonly unrecognized and misdiagnosed for several years. $^{3,4}$

While vulvar lichen sclerosus was first described over a century ago, uncertainty continues to exist around its pathogenesis, diagnosis and treatment. However, there 
have been great advances in diagnosis and progression of disease as well as investigation of new promising treatment modalities. The purpose of this review is to discuss the current understanding of etiopathogenesis, diagnostic challenges, and emerging treatments for vulvar lichen sclerosus.

\section{HISTORICAL PERSPECTIVE}

Weir described vulvar LS as "ichthyosis" in 1875. The classic histopathology of LS was first described by Darier in 1892. It was, thereafter, labeled as lichen sclerosus et atrophicus, as it was considered to be an atrophic lesion. Over the years, many terms were used to describe this condition such as kraurosis vulvae, hypoplastic dystrophy, Weiss flecken dermatose and leucoplakia. International society for the study of vulvovaginal disease (ISSVD) in 1976 discouraged the use of the term "kraurosis" and "leucoplakia," and since then, the term lichen sclerosus has been used. VLS was first documented in the pediatric population in $1901 .{ }^{3,5,6}$

Taussig in 1920 recommended surgery, i.e., vulvectomy, as the treatment of choice for kraurosis vulvae. Over the time various treatment modalities were described for VLS such as retinoids, hormonal therapy like testosterone and local steroid therapy. HLA serotyping and causal associations with infections like Borrelia were also reported in 1980 s. $^{6}$

\section{EPIDEMIOLOGY}

The exact prevalence of VLS is not known; the estimated rate of histologically proven VLS is $1.7 \%$ in general gynecology practice. The presentation is mainly bimodal, i.e., one in prepubertal girls (average age: 7.6 years) and the other in peri-and postmenopausal women (average age: 52.6 years), however, it can develop at any age. ${ }^{6}$ It can also occur during pregnancy and potentially affect pregnancy. In fact, recent studies demonstrate that over $50 \%$ of women with VLS have symptom onset before the age of $50 .^{7}$ However, the true prevalence is probably higher due to patient fear or embarrassment or under-recognition and underreporting of disease. ${ }^{8}$ The incidence of Vulvar lichen sclerosus is reportedly been rising. A recent study estimated that the incidence of histology-proven VLS in women rose from 7.4 to 14.6 per 100,000 woman-years between 1991 and 2011. The authors propose that this rise in incidence reflects an increased awareness of the condition. ${ }^{1,2}$

\section{ETIOPATHOGENESIS}

The etiology of VLS remains unknown, but several mechanisms have been studied for this noncontagious disease. Studies suggest a multifactorial origin as far as etiology is concerned, including a genetic, autoimmune, hormonal, and local and infectious background. ${ }^{6}$ Potential aetiological and risk factors are summarized in the Table 1 .
Genetic association to VLS has been shown in family and twin studies. In $22 \%$ of cases, family history of the disease affects sisters, mothers and daughters, and twin sisters including both homozygotic and heterozygotic twins. ${ }^{9}$ Women with VLS have an increased prevalence of certain human leucocyte antigens (HLAs) like HLA-DQ7, -DQ8, -DQ9, and -DR12 with $50 \%$ of adult females and $66 \%$ of prepubertal females expressing HLA-DQ7. ${ }^{10}$ Recently, epigenetic pathways have been implicated as causative or accelerant agents of disease, particularly miR-155, downstream targets of ECM1, galectin-7, p53, and epigenetic modifications to CDKN2A. ${ }^{11}$ There is a strong association between VLS and autoimmune disease in adults. Some specific antibodies have been reportedly, associated with VLS. Furthermore, some autoimmune diseases like diabetes mellitus type 1 , autoimmune thyroid diseases (Hashimoto thyroiditis and Graves' disease), psoriasis, alopecia areata, vitiligo, and pernicious anemia have been associated with this disease. ${ }^{12}$

Table 1: Potential etiological and risk factors of vulvar lichen sclerosus.

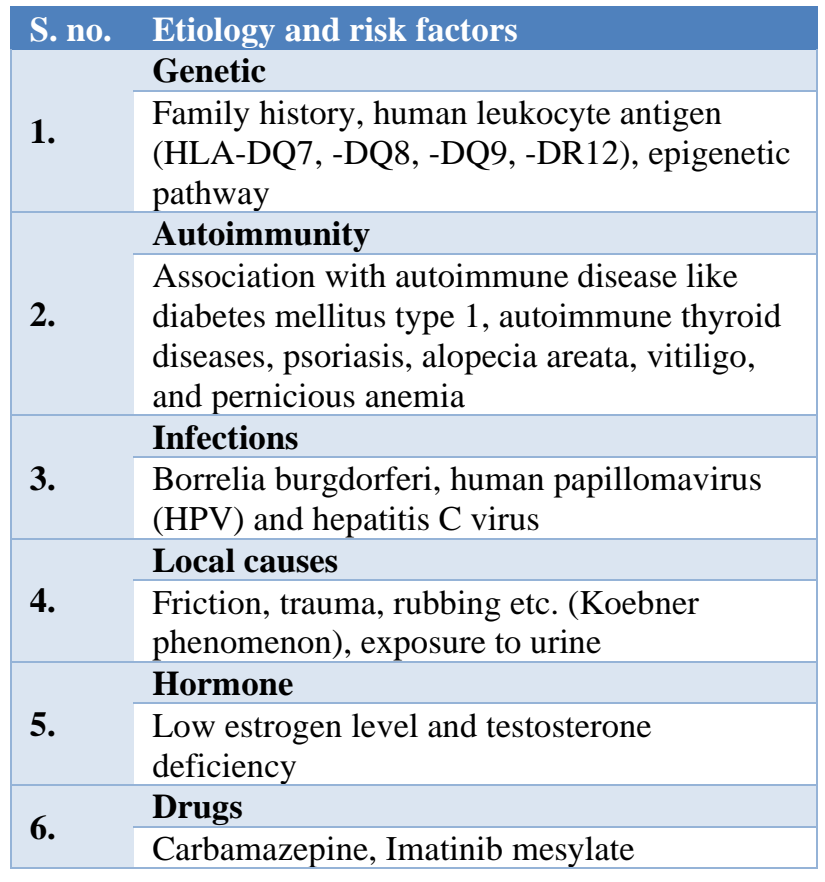

Involvement of these immune and genetic factors in LS pathology involve auto-immunogenic activation, sclerotic tissue formation and oxidative stress. ${ }^{11}$ MicroRNA-155 (miR-155) is involved in promoting T-helper type $\mathrm{I}\left(\mathrm{T}_{\mathrm{h}} 1\right)$ cytokines differentiation, upregulation of which causes inflammatory changes. ${ }^{13}$ Overexpression of miR-155 can disrupt suppressive function of regulatory $\mathrm{T}$ cells, triggering a loss of self-tolerance and promoting inflammation, and thereby inducing autoimmunity. ${ }^{11}$ Dysregulation due to overexpression of miR-155 is also associated with increased collagen synthesis, leading to sclerotic tissue formation. In addition, miR-155 inhibits tumor suppressor genes FOXO3 and CDKN1B, leading to even more collagen synthesis. ${ }^{14}$ Autoimmune mediated disruption of extracellular matrix protein 1 (ECM1), a 
scaffolding glycoprotein which acts as a "biological glue" at the dermal-epidermal junction, was thought to be the first target implicated in the development of LS. However, more recent studies indicate that autoimmunity to ECM1 alone is insufficient in explaining the pathogenesis of LS. ${ }^{11}$ Autoantibodies affect the regulatory binding of ECM1 to matrix metallopeptidase 9 (MMP9), which leads to overactive collagen synthesis, especially type V collagen, and disruption of focal basement membrane. ${ }^{15}$ Sclerotic tissue formation is also facilitated by dysregulation of p53 regulated protein galectin-7, which inhibits fibroblast growth and increases collagen synthesis. ${ }^{16}$ Oxidative stress due to upregulation of $\mathrm{T}$-helper type $\mathrm{I}\left(\mathrm{T}_{\mathrm{h}} 1\right)$ cytokines contribute to inactivation of tumor suppressor genes involving p53 and CDKN2A, leading to cell proliferation and transformation to malignancy. ${ }^{11}$ Clinical trial [NCT03561428] regarding identification of specific biomarkers like genes, protein, or glycoproteins is ongoing, which might facilitate the development of minimally invasive tests or screening tools for early detection of LS, as well as more specific tests for biopsybased tissue diagnosis. ${ }^{17}$

VLS is not contagious, but both bacterial and viral pathogens such as Borrelia burgdorferi, human papillomavirus (HPV) and hepatitis $\mathrm{C}$ virus have been implicated in its etiology but the evidence is contradictory. ${ }^{18}$ VLS can rarely be initiated through scarring or radiation. Local factors such as friction, trauma, or rubbing may cause Koebner phenomenon which is a well-known manifestation of VLS. ${ }^{3,6}$

Higher incidence of VLS in postmenopausal women and prepubertal girls with a low estrogen level suggest a hormonal influence, but a protective effect from estrogen has not been shown. ${ }^{3}$ Free testosterone and androstenediones were decreased in women with lichen sclerosus. A loss of androgen receptors in lesions caused by lichen sclerosus was demonstrated. ${ }^{19}$ Increased risk of VLS in Turner syndrome, but lack of variation with use of HRT and contraceptive pills, points to controversial role of hormones. The role of hormones has been put aside in recent years. ${ }^{6}$ Association of lichen sclerosus with certain drugs like carbamazepine, Imatinib mesylate also has been reported. ${ }^{20}$

\section{CLINICAL CHARACTERISTICS}

Most of the patient present with progressive pruritus but it may also present with vulvar pain and burning, dyspareunia, apareunia, anorgasmia, dysuria, fecal incontinence or genital bleeding. ${ }^{6}$ Progressive scarring may cause clitoral phimosis and narrowing of the vaginal introitus leading to dyspareunia. Tearing at the base of the fourchette may occur with intercourse due to progressive loss of tissue elasticity. ${ }^{1}$ Women with VLS have significantly lower score on a validated female genital selfimage scale (FGSIS), which have been found to correlate with sexual function. ${ }^{21}$ Dysuria and difficulty in voiding occur due to fusion of the labia minora over the urethra with advanced disease. Constipation and painful defecation may be a feature in case of perianal involvement but is rare in adult women. Painful defecation, anal fissures and rectal bleeding may require extensive gastrointestinal evaluation and sometimes hemorrhoidectomy or repair of an anal fissure. It may be asymptomatic in some women. ${ }^{3}$ In $20 \%$ of patients, other areas such as the upper trunk, axillae, buttock, and lateral thigh are involved. LS rarely involves the vaginal mucosa and cervix. ${ }^{1,6}$

On physical examination, initially vulva is bright, red and thick; labia minora may be edematous and partially resorbed. Hemorrhagic bullae may be also present in some cases. $^{6}$ Lichen sclerosus primarily involves non-hair bearing, inner areas of the vulva. ${ }^{22}$ With time, the vulva reveals ivory white atrophic plaques with a waxy texture or epidermal wrinkling ("cigarette paper" appearance), depigmentation or hyperpigmentation, ecchymoses and narrowing of the introitus. ${ }^{1}$ Involvement of labial, perineal, and perianal areas along with introital narrowing can lead to classic "keyhole" or "hourglass" or "figure of eight" appearance of lesion. In advanced stage, there is loss of labia minora, burying of the clitoris, obstruction of urinary outflow and distortion of vulvar and contiguous anatomy..$^{3,6}$

Advanced disease severely affects the quality of life (QoL) and is associated with increased risk of vulvar squamous cell carcinoma (SCC) with $4 \%-6.7 \%$ risk. ${ }^{1,2}$ Recent data show that the risk is low in the women appropriately treated and under follow-up. Longer duration of symptoms and loss of vulvar architecture increase the risk of the cancer. $^{23}$

\section{DIAGNOSIS}

Diagnosis of VLS is usually clinical, and is done by careful history taking and clinical examination. On the basis of clinical symptoms of erosions, hyperkeratosis, fissures, agglutination, stenosis, and atrophy, Naswa and Marfatia have administered a physician clinical score, and graded VLS as grade 1-no changes or normal, grade 2-moderate, and grade 3 -severe. The proper and timely usage of this clinical score helps in early diagnosis and prompt treatment of the disease and will help alleviate symptoms, prevent architectural damage, and reverse histologic changes. $^{24}$

A biopsy to confirm VLS prior to treatment is not necessary in typical presentation. However, diagnosis by histopathological examination is necessary with atypical features, uncertainty of diagnosis, concern for malignancy, or in failed response to treatment. Biopsy should ideally be collected without prior application of corticosteroid, or if necessary, after four weeks of therapy interruption, as treatment can resolve the pathognomonic histopathologic changes. ${ }^{1,25}$ Some specialists postulate that a biopsy should be taken in each case to confirm the clinical diagnosis. ${ }^{26}$ Biopsy should be performed from interface between 
normal and abnormal areas. It should also be taken from the hyperkeratotic areas and the erosions that do not improve with the treatment or sites with the altered pigmentation. ${ }^{6}$ A biopsy is usually taken under local anesthesia and is generally well tolerated while in the children it is not done, because in the ano-genital area this can be a very traumatic experience. When the clinical and histological findings differ, repeat biopsy must be performed. ${ }^{20}$ Characteristic histopathological changes generally include hyperkeratosis of the epidermis, epidermal atrophy with loss of rete ridges, homogenization of the collagen in the upper dermis, and a lichenoid (bandlike) inflammatory infiltrate in the dermis. ${ }^{1}$ Epidermal hyperplasia or dysplasia on vulvar biopsy is associated with increased risk of the malignant transformation. Overexpression of wild-type p53 is also associated with the increased cancer risk as it is an HPV-associated increase in the p16INK4A. ${ }^{3}$

Dermoscopy can aid in clinical diagnosis of VLS and its characteristic pattern include sparse dotted vessels; patchy, structureless areas, whitish to white-yellow to pinkwhitish color over a diffuse whitish background; grey-blue dots, usually with a characteristic peppered arrangement, corresponding to dermal melanophages; comedo-like openings and scales; as well as peculiar structures like ice slivers. ${ }^{3,27}$

Assessment should also include workup for autoimmune diseases such as type 1 diabetes mellitus, thyroid disease, scleroderma, and rheumatoid arthritis as there is an association between VLS and autoimmune diseases. The assessment should be clinical, and investigations should be performed when indicated.

Differential diagnosis of VLS include lichen planus (erosive/mucosal form), vitiligo, eczema, psoriasis, autoimmune blistering dermatosis, candida infection localized scleroderma, leukoplakia, vitiligo, immunobullous disorders such as cicatricial pemphigoid, cutaneous patch of Lyme's disease and squamous cell hyperplasia. Ecchymotic and bleeding lesions may lead to suspicion of sexual abuse in children. ${ }^{6,20}$

\section{TREATMENT}

There is no definitive cure for VLS. The major problem for patients is genital itching and pain. The goal of treatment is to alleviate symptoms of itching and pain, prevent anatomic changes due to scarring, and possibly prevent malignant transformation. ${ }^{1}$ Management of pruritus vulvae can be divided into four stages: (1) identification of underlying disease; (2) restoration of the skin barrier function; (3) reduction of any inflammatory complication; and (4) elimination of the itch-scratch cycle by psychological methods. Asymptomatic VLS cases should be treated too. ${ }^{28}$ There are a number of treatment options that have been tried to achieve the remission and prevent progression of the disease, are described below in the Table 2.
Table 2: Treatment modalities for vulvar lichen sclerosus.

\begin{tabular}{|ll|}
\hline S. & Treatment of vulvar lichen sclerosus \\
no. & Ultrapotent topical corticosteroids \\
\hline 1. & Topical calcineurin inhibitors \\
\hline 2. & $\begin{array}{l}\text { Hormonal therapy (Estrogen, topical } \\
\text { androgens, progesterone) }\end{array}$ \\
\hline 3. & Topical retinoids \\
\hline 5. & Other local pharmacologic agents \\
\hline 6. & Intralesional therapy \\
\hline 7. & Role of emollients and herbal therapy \\
\hline 8. & $\begin{array}{l}\text { Energy-based modalities-Photodynamic } \\
\text { therapy (PDT), high intensity focused }\end{array}$ \\
\hline 9. & Cryotherapy \\
\hline 10. & Phototherapy \\
\hline 11. & $\begin{array}{l}\text { Adipose-derived stem cells and platelet rich } \\
\text { plasma therapy }\end{array}$ \\
\hline 12. & Systemic therapy \\
\hline 13. & Diet \\
\hline 14. & Surgical management \\
\hline
\end{tabular}

\section{Ultrapotent topical corticosteroids}

The gold standard treatment for VLS is ultrapotent topical corticosteroids (TCSs), most commonly clobetasol propionate $(0.05 \%)$ ointment. It significantly reduces symptoms and improves skin characteristics. Clobetasol or halobetasol propionate $0.05 \%$ ointment is a class I super potent topical steroid which suppresses mitosis, increases the synthesis of proteins, decreases inflammation and cause vasoconstriction. ${ }^{3}$ According to the 2018 British association of dermatologists (BAD) guidelines, recommended dose is a half-fingertip unit (approximately $0.5 \mathrm{gm}$ ) applied to the affected area once daily for 1 month, then every other day for 1 month, and then twice weekly for a third month. Once symptoms are controlled, topical steroid may be applied as needed for recurrent symptoms. ${ }^{25}$ The 2015 European guidelines recommend daily application for the first 3 months, then individualized maintenance ranging from 1-2 times per month to 2-3 times per week. ${ }^{29}$ Ointments are more potent than creams. Creams may contain alcohols or preservatives that are much more likely to cause burning, stinging, and/or irritation, particularly of the inflamed/fissured mucosa, and hence, ointments are preferred over creams. Hypopigmentation and dermal atrophy may occur rarely with topical steroid use, particularly to keratinized skin. ${ }^{30}$ Potent steroids in pulse dosing can be given, i.e., 2 consecutive days/week with mild steroids or simple emollients in between to reduce the side effect. ${ }^{3}$ The lesspotent steroids like mometasone furoate $(0.1 \%)$ and triamcinolone are also found to be effective. Clobetasol propionate ointment $0.05 \%$ is more effective in VLS compared to topical tacrolimus $0.1 \%$, topical testosterone $2 \%$, and phototherapy. Optimal and appropriate topical corticosteroid treatment decreases the risk of malignant transformation and progression of vulvar scarring. ${ }^{1}$ TCS 
are also the mainstay of treatment of VLS in children and pregnant patient. However, these drugs should be used cautiously keeping risk benefit ratio in mind. ${ }^{29,30}$

Some studies have suggested use of different corticosteroids on the basis of severity of disease. Severity was assessed by the degree of hyperkeratosis of the lesion. Very severe disease should be treated with clobetasol propionate ointment, moderate to severe disease with betamethasone dipropionate ointment, mild disease with methylprednisolone aceponate ointment, and very mild "burnt out" disease with hydrocortisone ointment. However, Cochrane Reviews states that potency and dose of topical steroid therapy for treatment of VLS needs further evaluation and research. After review of various studies, we therefore suggest usage of clobetasone propionate ointment $(0.05 \%)$ for treatment of any degree of VLS. ${ }^{30}$

\section{Topical calcineurin inhibitors (TCIs)}

Tacrolimus $(0.1 \%)$ and pimecrolimus $(1 \%)$ are second choice treatment options. ${ }^{20}$ They are not as effective as potent topical corticosteroids for VLS and may be useful as alternative treatment options. These drugs have a pronounced anti-inflammatory, an immunomodulatory and a weakly pronounced immunosuppressive effect. ${ }^{28}$ They reduce itching and inflammation by blocking T-cell activation and suppressing the release of cytokines from $\mathrm{T}$ cells. They do not inhibit collagen synthesis so they do not cause dermal atrophy, which may be especially useful in pediatric patients. ${ }^{1,3}$ They are effective when used daily or twice daily in children and adults with partial to complete remission ranging from six weeks to 10 months of treatment. These drugs are also used with topical corticosteroids as combination therapy and bridging therapy. Side effects of TCIs include stinging and burning so often discontinued by patients. There is also concerns about the increased risk of neoplasia, especially with longterm use. ${ }^{28,30}$

\section{Hormonal therapy}

Estrogen is an effective treatment for postmenopausal vulvovaginal dryness and atrophy but not used as a primary therapy for VLS. ${ }^{3}$ Topical androgens like testosterone propionate cream (2\%), dihydrotestosterone (DHT) cream (2\%), and progesterone (2\%) were mainstay of treatment for decades and were reported to induce remission of VLS. But now a days their use has been stopped due to worse results compared to those of ultrapotent topical corticosteroids and their side effects (systemic absorption and virilizing effects in female patients). ${ }^{28,30}$

\section{Topical retinoids}

Topical tretinoin $(0.025 \%)$ and tazarotene $(0.01 \%)$ may represent a useful alternative option to corticosteroids in the treatment of active VLS. They have anti-inflammatory properties and reduce hyper-keratinization. They act by downregulation of fibroblast function. Short-contact therapy is used in which the gel is initially applied for 15 min and washed off. Every 2-3 weeks, application time is increased by 15 min until therapeutic effects are noted. However, these are not commonly used now a days. Also, contraindicated in pregnancy because of teratogenic effect. $^{28,30}$

\section{Other local pharmacologic agents}

Topical oxatomide helps relieve pruritus through its antihistaminic effects, but the course of disease is not affected. ${ }^{3}$

Therapy with TheresienOl (herbal oil) twice daily for two to three months has been found to be effective in relieving clinical symptoms of VLS. ${ }^{31}$

Topical icilin, which is a transient receptor potential channel subfamily M (melastatin) member 8 (TRPM8) agonist has been studied as pruritus relieving agent in VLS. $^{3}$

Topical avocado and soybean extracts has been suggested as alternative treatments for mild-to-moderate VLS in patients wishing to avoid corticosteroids. Their topical and dietary supplements exert anti-inflammatory, antifibrotic, emollient and lenitive actions. ${ }^{3}$

Local application of human fibroblast lysate cream, containing anti-inflammatory cytokines as well as woundhealing growth factors, has also been found to be useful in treatment.

Antibiotic like penicillin or cephalosporins has been tried in VLS with secondary infection. They help in relieving symptoms like pain, pruritus and burning. ${ }^{30}$

\section{Intra-lesional therapy}

Intra-lesional injection of triamcinolone acetonide has been proposed as an alternative treatment to topical treatment of thick LS because there is lack of response to a steroid cream or ointment due to poor penetrance. It is given as subdermal injection in a dose of $20 \mathrm{mg}$ once a month for two to three months. It is also used in combination therapy with topical corticosteroid therapy for the lesions not responding to single therapy. Intralesional injection of adalimumab has also found to be effective. ${ }^{3,30}$

\section{Role of emollients and herbal therapy}

Emollients or moisturizers should be an integral part of treatment of VLS. They keep the skin soft and protect from cracks in VLS patients. Application of moisturizers or paraffin prevents skin contact with urine, and reduce symptoms. They provide relief from pain when used during sexual intercourse. Being safe and inexpensive, 
therapy with a moisturizing cream can be used for longer time to maintain the symptom relief induced by TCS. Use of soap should be substituted with emollients. Use of abrasive towels, special intimate care products and tight clothing should be avoided. ${ }^{20,30}$ Silk briefs should be preferred over cotton for symptomatic relief. ${ }^{3}$

Herbal therapies with agents like chamomile, coconut oil, shea butter, aloe vera, calendula, arnica, avocado, etc. have been used in VLS with variable results. The fact that whether they act as emollient or have any therapeutic potential is controversial. Further studies are required to prove their efficacy in treatment of VLS. ${ }^{30}$

\section{Energy-based modalities}

Three energy-based modalities have recently been studied for the treatment of LS: photodynamic therapy (PDT), High-intensity focused ultrasound (HIFU) and laser therapy. These methods have been described in few patients and cannot be routinely applied.

\section{Photodynamic therapy (PDT) using a photosensitizer}

PDT produces intracellular reactive oxygen radicals from uptake of the exogenous-photosensitizing agent or via endogenous production which target cells that play a role in inflammation and fibrosis, with limited damage to healthy tissue. Good symptomatic improvement (itching, burning and pain) has been seen in patients treated with PDT with the use of topical 10\% 5-ALA cream (5aminolaevulinic acid). ALA-PDT is well-tolerated and shows longer remission duration and a higher complete response rate than clobetasol propionate. Pain is main adverse reaction in ALA-PDT. Steroids can be used as a palliative treatment for patients who relapse after ALAPDT. ${ }^{1,30}$

One study reported, significant improvement with the use of topical 5-ALA with argon laser light. Another study demonstrated good symptomatic benefit with aminolevulinic acid-PDT using a bioadhesive patch. ${ }^{3,30}$ There is an ongoing trial on the Rivelin ${ }^{\circledR}$ plain patch, which works by forming a protective barrier over a lesion, hence protecting it from further irritation for longer duration. ${ }^{30}$ Although PDT seems promising, additional research is needed to determine efficacy of this treatment modality.

\section{High-intensity focused ultrasound (HIFU)}

HIFU stimulates cell proliferation, protein synthesis, and revascularization, thereby accelerating tissue reconstruction. It decreases inflammation and shows significant symptomatic improvement in patients of VLS. However, side effects like skin burn and blistering can be seen in some cases. A recent study found that treatment with HIFU led to "curative effects" on histology compared to the topical corticosteroid group. ${ }^{1}$ Additional research is needed to support adoption of this promising treatment modality.

\section{Laser}

Ablative lasers (fractional carbon dioxide laser) and nonablative \{pulsed dye and erbium-doped yttrium aluminum garnet lasers (Er:YAG)\} lasers have been reported to benefit VLS symptomatically but did not stop the disease recurring. Non-ablative ones, unlike ablative lasers, have no side effects such as pain, discomfort, scars, infections, pigmentation or bleeding. ${ }^{28,30}$ There is an ongoing trial on the MonaLisa touch laser (DEKA Laser, Florence, Italy) looking at the efficacy and safety of the micro-ablative fractional $\mathrm{CO}_{2}$ laser treatment $\left(\mathrm{FxCO}_{2}\right)$ for VLS (Trial no. NCT03665584). ${ }^{30}$ In addition, there is also an ongoing trial comparing the safety and efficacy of $\mathrm{FxCO}_{2}$ laser therapy to topical clobetasol treatment of VLS [NCT02573883]. ${ }^{1}$

\section{Cryotherapy}

Cryotherapy may be used as a second-line therapy option in the treatment of long-standing VLS resistant to medical therapy. Temperature of $-186^{\circ} \mathrm{C}$ under general anesthesia has been used. Contact method with one freeze-thaw cycle per lesion and session combined with intra-lesional steroid injection (triamcinolone acetonide) has been used successfully. However, limiting factors of cryotherapy are a relatively high number of relapses, a long post-operative healing time, and a long time for pain-free status after cryotherapy. ${ }^{3,30}$

\section{Phototherapy}

Treatment with phototherapy using narrow-band ultraviolet B and ultraviolet A1 have been found to show significant improvement in VLS. However, these improvements are inferior to that of the current gold standard treatment with topical high-potent corticosteroids and hence, these options should be reserved for refractory cases as second-line therapy. 3,30

\section{Adipose-derived stem cells and platelet-rich plasma therapy}

Adipose-derived stem cells are purported to be able to restore and regenerate damaged tissue as in VLS. They act as regenerative agent and also have anti-inflammatory and immunomodulatory properties. They inhibit fibrosis, promote healing, and remodel the extracellular matrix. Similarly, platelet-rich plasma (PRP) therapy promotes healing process by its regenerative potential as it has tissue factors for cell growth, migration, repair, proliferation, and regeneration. ${ }^{1,32}$

\section{Systemic therapy}

Systemic treatment is occasionally indicated in refractory cases. Retinoids like isotretinoin, etretinate and acitretin appear to reduce connective tissue degeneration in VLS. 
However, the use of these agents is limited by potentially harmful side effects including cheilitis, xerosis, teratogenicity, elevated liver enzymes, hypertriglyceridemia, abdominal pain, and alopecia. Hydroxyzine or doxepin may be given to stop nighttime pruritus. Hydroxychloroquine and hydroxycarbamide (hydroxyurea) have been reported to be effective in widespread genital and extragenital LS with conflicting results. $^{3,20,30}$

Subcutaneous triamcinolone in the vulvar tissue has been attempted in cases of worse pruritus. Cycloferon, cyclosporine and potassium para-aminobenzoate have also been tried in VLS with promising results. ${ }^{3,30}$

\section{Diet}

Pork consumption and other foods which are high in oxalate like spinach, canned and boxed food items, potatoes, nuts, cocoa, beets, turnip, etc. should be avoided in VLS as these are associated with worsening of symptoms. Dietary supplements rich in vitamin E, calcium and para-aminobenzoic acid are associated with improvement of symptoms in VLS. ${ }^{3,30}$

\section{Surgical management}

Surgical intervention in VLS should be reserved for patients with disease refractory to medical management, to correct irreversible scarring, adhesions and, micturition difficulties or sexual dysfunction caused by the subsequent anatomical changes, or to prevent the development of invasive carcinoma of the vulva. Surgery is done to release a buried clitoris, to separate fused labia, or to widen a narrowed introitus in the case of pain or sexual dysfunction. V-Y advancement flap is an effective method for the reconstruction of perineal region. This technique will allow the expansion of vaginal orifice with good cosmetic results and rapid healing after surgery. ${ }^{3,22,30} \mathrm{~A}$ minimally invasive surgical procedure can treat clitoral phimosis and improve clitoral sensitivity and ability to achieve orgasm. ${ }^{1}$ Recurrent VLS following simple excision or adhesiolysis may successfully be treated with wider excision and reconstruction with split skin graft (SSG). More aggressive surgical treatment like vulvectomy is reserved for women with malignancy. ${ }^{30,33}$ However, these techniques can be debilitating, prone to recurrence, and lead to incomplete resolution of symptoms with low satisfaction. ${ }^{22}$

Surgical separation of tissue can often result in adhesions and increased scarring known as the Koebner phenomenon which can be prevented by application of clobetasol $0.05 \%$ ointment daily to the surgical site until well healed. ${ }^{34}$ Reformation of adhesions can also be prevented by resection of a fragment of the clitoral hood in the shape of a tricorn. ${ }^{35}$ Oxidized regenerated cellulose has also been studied to prevent recurrence of adhesions. ${ }^{36}$ More studies are required as far as surgical management of VLS is concerned.

\section{Prognosis and follow-up}

Lichen sclerosus is typically a lifelong disease. When VLS begins in childhood, permanent remission in puberty cannot be expected with certainty. ${ }^{20}$ However, VLS has no associated increased mortality except malignancy and progressive scarring. QoL is affected as it results in severe sexual dysfunction. ${ }^{3}$ So, regular follow-up is necessary to prevent recurrence and complications. Follow-up may be done every three to six months for the first two years and then at least yearly to ensure adequacy of treatment and encourage compliance. Long-term follow-up is recommended who have persistent complaints, thickened skin, or history of neoplastic lesion. ${ }^{30}$

\section{Patient counseling}

Most of the women with VLS or any vulvovaginal condition experience feelings of isolation, hopelessness, depression, anxiety, anger and low self-image. Some women are unable to continue working, any physical activity or sexual relations. So, psychological counseling is very much needed in such patients. ${ }^{3}$

Patients should be counseled for self-care and to maintain vulval hygiene. Use of soap should be substituted with emollients. Carefully dry the area after passing urine to reduce the contact of urine or using a barrier cream like moisturizer or soft paraffin to protect skin from exposure to urine. Lubricants and, if required, vaginal dilators may aid if intercourse is painful. Light, soft, airy and frictionless silk briefs should be recommended for symptomatic relief. 3,30

Patients should be counseled for regular self-examination and if any skin change develops which does not respond to steroid creams, or there is any skin thickening, soreness, or ulceration lasting more than 2 weeks, consult without delay. Patients should be advised against smoking as it increases the risk of vulvar cancer. It is important to emphasize that VLS needs to be treated even if asymptomatic to prevent scarring leading to sexual and urinary problems. ${ }^{3,30}$

\section{Future modality of treatment}

Recent studies indicate that the platelet-rich plasma (PRP) and stem cells used along with autologous fat transfer deserves a special mention as a novel technique in the management of VLS, which needs additional research. Future research should also focus on elucidating the aetiopathogenesis and true prevalence of VLS, which could give rise to earlier and more specific management options.

\section{CONCLUSION}

VLS is a chronic debilitating disease, which affects women of all ages. There is often a delay in diagnosis of VLS due to lack of awareness in patients as well as physicians. Highly potent topical corticosteroids are the 
first-line treatment. Most of the second-line therapies are in experimental phases, and further data is required.

Early detection followed by prompt and lasting treatment, as well as patient support, timely intervention and regular follow up is essential to prevent complications of VLS and to improve the quality of life.

Funding: No funding sources

Conflict of interest: None declared

Ethical approval: Not required

\section{REFERENCES}

1. Krapf JM, Mitchell L, Holton MA, Goldstein AT. Vulvar Lichen Sclerosus: Current Perspectives. Int J Women's Health 2020;12:11-20.

2. Bleeker MCG, Visser PJ, Overbeek LIH, Beurden MV, Berkhof J. Lichen sclerosus: incidence and risk of vulvar squamous cell carcinoma. Cancer Epidemiol Biomarkers Prevention 2016;25:1224-30.

3. Nair PA. Vulvar Lichen Sclerosus et Atrophicus. J Mid-Life Health. 2017;8:55-62.

4. Schlosser BJ, Mirowski GW. Lichen sclerosus and lichen planus in women and girls. Clin Obstet Gynecol. 2015;58:125-142.

5. Funaro D. Lichen sclerosus: A review and practical approach. Dermatol Ther. 2004;17:28-37.

6. Singh N, Ghatage P. Etiology, clinical features, and diagnosis of vulvar lichen sclerosus: a scoping review. Obstet Gynecol Int. 2020;2020:7480754.

7. Christmann-Schmid C, Hediger M, Gr€oger S, Krebs $\mathrm{J}$, G€unthert AR. Vulvar lichen sclerosus in women is associated with lower urinary tract symptoms. Int Urogynecol J. 2018;29(2):217-21.

8. Halonen P, Jakobsson M, Heikinheimo O, Gissler M, Pukkala E. Incidence of lichen sclerosus and subsequent causes of death: a nationwide Finnish register study. BJOG. 2020;127:814-9.

9. Van de Nieuwenhof HP, Van der Avoort I, De Hullu JA. Review of squamous premalignant vulvar lesions. Crit Rev Oncol Hematol. 2008;68:131-56.

10. Gao X-H, Barnardo MCMN, Winsey S, Ahmad T, Cook J, Agudelo JD. The association between HLA DR, DQ antigens, and vulval lichen sclerosus in the UK: HLA DRB112 and its associated DRB112/DQB10301/04/09/010 haplotype confers susceptibility to vulval lichen sclerosus, and HLA DRB10301/04 and its associated DRB10301/04/DQB10201/02/03 haplotype protects from vulval lichen sclerosus. J Invest Dermatol 2005;125:895-9.

11. Tran DA, Tan X, Macri CJ, Goldstein AT, Fu SW. Lichen Sclerosus: an auto-immuno-pathogenic and genomic enigma with emerging genetic and immune targets. Int J Biol Sci. 2019;15:1429-39.

12. Kazandi M, Sahin C, Terek MC. Borrelia in granuloma annulare, morphea and lichen sclerosus: a PCR-based study and review of the literature. J cutaneous Pathol. 2010;37:571-7.
13. Terlou A, Santegoets LAM, Van der Meijden WI, Heijmans-Antonissen C, Swagemakers SMA, Van der Spek P. An autoimmune phenotype in vulvar lichen sclerosus and lichen planus: a Th1 response and high levels of MicroRNA-155. J Invest Dermatol 2012;132:658-66.

14. Ren L, Zhao Y, Huo X, Wu X. MiR-155-5p promotes fibroblast cell proliferation and inhibits FOXO signaling pathway in vulvar lichen sclerosis by targeting FOXO3 and CDKN1B. Gene. 2018;653:4350.

15. Godoy CAP, Teodoro WR, Velosa APP, Garippo AL, Eher EM, Parra ER. Unusual remodeling of the hyalinization band in vulval lichen sclerosus by type V collagen and ECM 1 protein. Clinics. 2015;70:35662.

16. Zhao Y, Zhao S, Li H, Qin X, Wu X. Expression of galectin-7 in vulvar lichen sclerosus and its effect on dermal fibroblasts. Oncol Lett. 2018;16:2559-64.

17. Goldstein AT. Biomarkers of lichen sclerosus NCT03561428. Clinicaltrials.gov. June 2019. Available https://clinicaltrials.gov/ct2/show/NCT03561428. Accessed on January 3, 2020.

18. Kazandi M, Sahin C, Terek MC, Cirpan T, Oztekin K. Clinical evaluation of vulvar lichen sclerosus: case series. Euro J Gynaecol Oncol. 2010;31:555-8.

19. Taylor AH, Guzail M, Al-Azzawi F. Differential expression of oestrogen receptor isoforms and androgen receptor in the normal vulva and vagina compared with vulval lichen sclerosus and chronic vaginitis. Br J Dermatol. 2008;158:319-28.

20. Kirtschig G. Lichen sclerosus-presentation, diagnosis and management. Dtsch Arztebl Int. 2016;113:33743.

21. Hodges KR, Wiener CE, Vyas AS, Turrentine MA. The female genital self-image scale in adult women with vulvar lichen sclerosus. J Low Genit Tract Dis. 2019;23:210-3.

22. Gujral S, Coelho J, Chummun S, Watts A. Management of Vulval Lichen Sclerosus-The Role of the Plastic Surgeon. Availabe at: www.ePlasty.com. Accessed on April 24, 2019.

23. Micheletti L, Preti M, Radici G. Vulvar lichen sclerosus and neoplastic transformation. J Lower Genital Tract Disease. 2016;20:180-3.

24. Naswa S, Marfatia YS. Physician-administered clinical score of vulvar lichen sclerosus: A study of 36 cases. Indian J Sex Transm Dis. 2015;36:174-7.

25. Lewis FM, Tatnall FM, Velangi SS, Bunker CB, Kumar A, Brackenbury F. British association of dermatologist's guidelines for the management of lichen sclerosus 2018. Br J Dermatol. 2018;178:8234.

26. Lee A, Bradford J, Fischer G. Long-term management of adult vulval lichen sclerosus. A prospective cohort study of 507 women. JAMA Dermatol. 2015;151(10):1061-7.

27. Borghi A, Corazza M, Minghetti S, Bianchini E, Virgili A. Dermoscopic features of vulvar lichen 
sclerosus in the setting of a prospective cohort of patients: new observations. Dermatology. 2016;232:71-7.

28. Yordanov A, Tantchev L, Kostov S, Slavchev S, Strashilov S, Vasileva P. Vulvar leukoplakia: therapeutic options. Menopause Rev. 2020;19:135-9.

29. Kirtschig G, Becker K, Günthert A, Jasaitiene D, Cooper S, Chi C-C. Evidence-based (S3) Guideline on (anogenital) Lichen sclerosus. J Eur Acad Dermatol Venereol. 2015;29(10):e1-43.

30. Singh N, Mishra N, Ghatage P. Treatment Options in Vulvar Lichen Sclerosus: A Scoping Review. Cureus. 2021;13:e13527.

31. Yordanov AD, Vasileva PP, Strashilov SA, Konsoulova A. Treatment of vulvar leukoplakia with TheresienOL- A new opportunity. Arch Balkan Med Union. 2019;54:753-8.

32. Eshtiaghi P, Sadownik LA. Fact or fiction? Adiposederived stem cells and platelet-rich plasma for the treatment of vulvar lichen sclerosus. J Low Genit Tract Dis. 2019;23:65-70.
33. Rangatchew F, Knudsen J, Thomsen MV, Drzewiecki KT. Surgical treatment of disabling conditions caused by anogenital lichen sclerosus in women: an account of surgical procedures and results, including patient satisfaction, benefits, and improvements in healthrelated quality of life. J Plast Reconstr Aesthet Surg. 2017;60:501-8.

34. King M, Rubin R, Goldstein AT. Current uses of surgery in the treatment of genital pain. Current Sexual Health Rep. 2014;6:252-8.

35. Rouzier R, Haddad B, Deyrolle C, Pelisse M, MoyalBarracco M, Paniel BJ. Perineoplasty for the treatment of introital stenosis related to vulvar lichen sclerosus. Am J Obstet Gynecol. 2002;186:49-52.

36. Wyman AM, Lafranchise E, Lynch C. External use of an oxidized regenerated cellulose agent in the treatment of labial agglutination in postmenopausal women. Obstet Gynecol. 2018;132:1238-40.

Cite this article as: Kumari A, Singh PK, Rohatgi R. Vulvar lichen sclerosus: recent advances in understanding and management. Int J Reprod Contracept Obstet Gynecol 2021;10:4029-37. 